\title{
Carcinoid syndrome caused by a serotonin-secreting pituitary tumour
}

\author{
Louise A Lynggård, Eigil Husted Nielsen and Peter Laurberg \\ Department of Endocrinology, Aalborg University Hospital, Hobrovej 18-22, DK-9000 Aalborg, Denmark
}

Correspondence

should be addressed

to P Laurberg

Email

peter.laurberg@rn.dk

\begin{abstract}
Background: Neuroendocrine tumours are most frequently located in the gastrointestinal organ system or in the lungs, but they may occasionally be found in other organs.

Case: We describe a 56-year-old woman suffering from a carcinoid syndrome caused by a large serotonin-secreting pituitary tumour. She had suffered for years from episodes of palpitations, dyspnoea and flushing. Cardiac disease had been suspected, which delayed the diagnosis, until blood tests revealed elevated serotonin and chromogranin A in plasma. The somatostatin receptor (SSR) scintigraphy showed a single-positive focus in the region of the pituitary gland and MRI showed a corresponding intra- and suprasellar heterogeneous mass. After pre-treatment with octreotide leading to symptomatic improvement, the patient underwent trans-cranial surgery with removal of the tumour. This led to a clinical improvement and to a normalisation of SSR scintigraphy, as well as serotonin and chromogranin A levels.

Conclusion: To our knowledge, this is the first reported case of a serotonin-secreting tumour with a primary location in the pituitary.
\end{abstract}

\section{Introduction}

The carcinoid syndrome consists of a variety of symptoms which typically include episodes of dry flushing with or without palpitations, diarrhoea and intermittent abdominal pain, whereas the severe state of a carcinoid crisis is characterised by distinctive flushing, bronchospasm, tachycardia and a widely and rapidly fluctuating blood pressure (1). Only a few patients have all the symptoms $(1,2)$. Consumption of food, alcohol intake and stress are well-known provocative factors, particularly of flushing and blood pressure alterations (2).

Differential diagnoses are numerous, including physiological and psychological stress, drug effects, primary cardiac arrhythmia, thyrotoxicosis and various other diseases. This often delays the diagnosis. Average diagnostic delay is up to 5-7 years from the initial onset of symptoms (3).

The syndrome is caused by the release of serotonin (5-hydroxytryptamin) and other substances from a neuroendocrine tumour (NET), most often located in the gut or in the lungs.

\section{Case}

A 56-year-old previously healthy Caucasian woman was referred for evaluation of a suspected NET syndrome.

Retrospectively, the patient began to experience small episodes of flushing nearly 15 years earlier, and this gradually worsened over the years. During the last 3-4 years her life had been dominated by increasingly frequent attacks of palpitations and tachycardia accompanied by dyspnoea, sensation of choking, dizziness, a buzzing sensation in the ears, visual disturbance, sweats, nausea, near-fainting and flushing, followed by hours of strong fatigue. These episodes might last from a few minutes to 2 days and they could be provoked by rapid movements or by bending the head towards the floor. There was no (c) 2014 European Society of Endocrinology Printed in Great Britain
Published by Bioscientifica Ltd. 

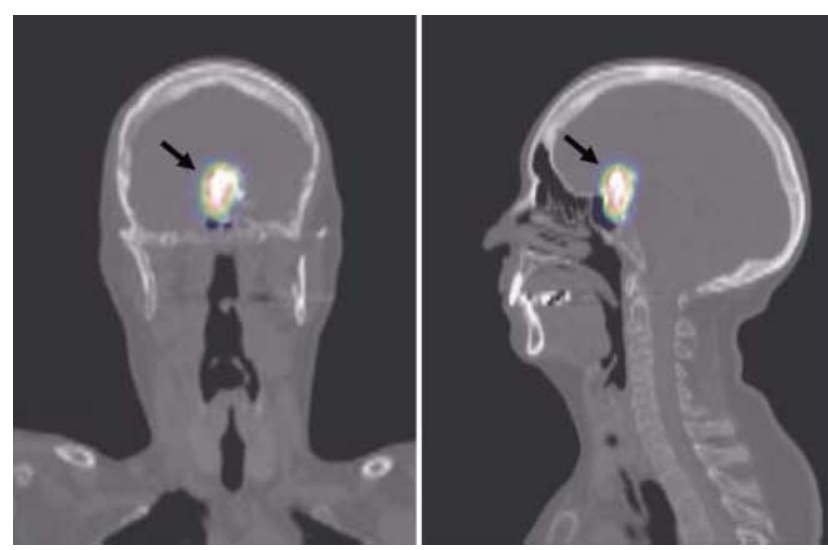

\section{Figure 1}

Somatostatin receptor scintigraphy with 111-indium-octreotide revealing an octreotide-positive focus (arrow) in the fossa hypophysialis.

diarrhoea or abdominal pain and the symptoms were not related to alcohol intake.

Due to the cardiac symptoms, she had been repeatedly investigated by cardiologists, and several arrhythmias such as pseudoventricular tachycardia, ectopic atrial tachycardia and sinus-inappropriate tachycardia had been observed after acute admissions. Radiofrequency ablation and therapy with several anti-arrhythmic drugs had insignificant effects.

A new diagnostic work-up revealed a clearly elevated plasma serotonin $(258 \mathrm{nmol} / \mathrm{l}$; ref $<30)$, a slightly elevated plasma chromogranin A (165 pmol/l; ref 30-130) and normal levels of 24-h urinary 5-Hydroxyindoleacetic acid (5-HIAA) excretion ( $16 \mu \mathrm{mol} /$ day; ref $10-40)$, results compatible with a carcinoid syndrome (1).

A somatostatin receptor (SSR) scintigraphy with 111indium-octreotide was performed. This revealed an isolated large abnormal octreotide-positive focus in the fossa hypophysialis (Fig. 1), with no other traceable octreotide-/SSR-positive foci in the abdominal organs, gastrointestinal tract or thorax. MRI of the pituitary region showed a $4 \times 3 \mathrm{~cm}$ large heterogeneous intra- and suprasellar tumour mass (Fig. 2), which was found highly calcified by CT-scan. Visual field examination showed a small left visual field reduction. Endocrinological evaluation revealed a normal pituitary function for age. Plasma metanephrin and normetanephrin were in the normal range. Echocardiography performed just before surgery showed no signs of valvular or other structural or functional abnormalities.
To stabilise the clinical condition, the patient was immediately given octreotide LAR $20 \mathrm{mg}$ i.m. This had a good symptomatic effect and the injection was repeated after 3 weeks. Five days later the patient underwent uncomplicated trans-cranial surgery with removal of the tumour.

The histological examination of the resected mass, including light microscopy and immunohistochemistry, revealed a calcified solid tumour with benign characteristics and consisting of small relatively uniform cells with sparse chromofobic cytoplasm. Practically, all cells showed positive reaction for glycoprotein hormone $\alpha$ subunits, whereas there were no positivities for thyroidstimulating hormone, adrenocorticotrophic hormone, betaendorfin, follicle-stimulating hormone, growth hormone $(\mathrm{GH})$, luteinizing hormone or prolactin. There was no reaction for serotonin, and CDX2 (marker of GI origin of NET (4)) and TTFI (marker of 'foregut origin' (4))

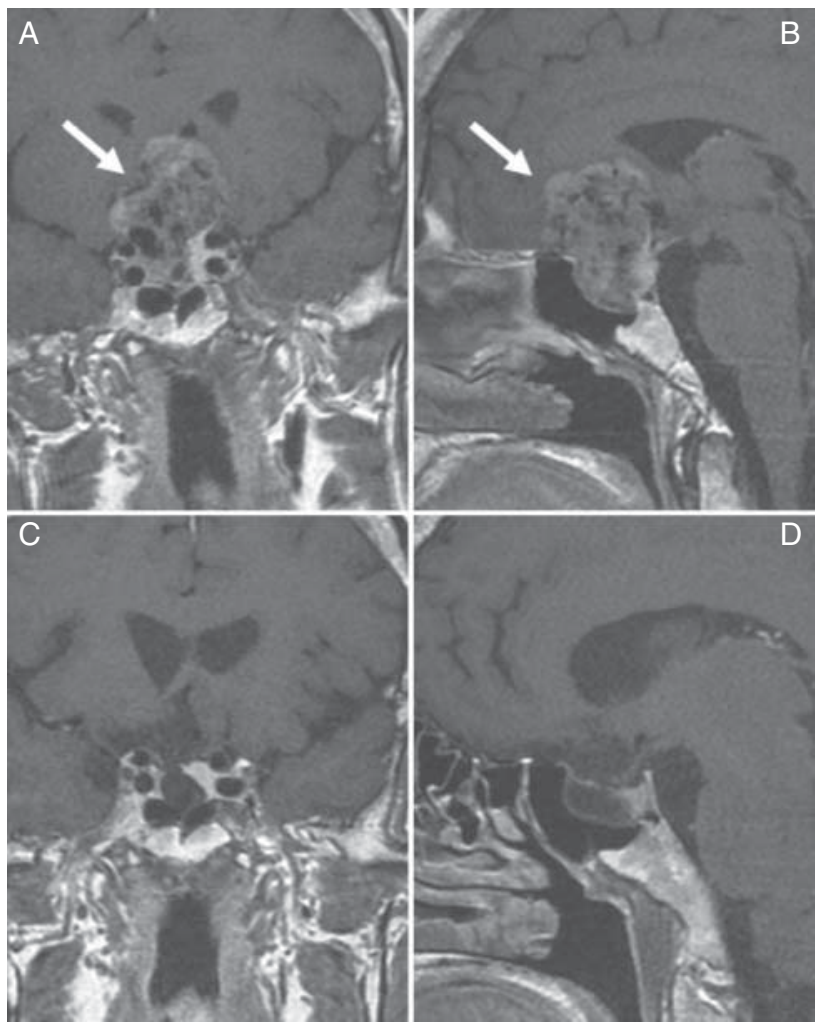

\section{Figure 2}

Preoperative coronal (A) and sagittal (B) MRI scans with gadovist showing a $4 \times 3 \mathrm{~cm}$ heterogeneous intra- and suprasellar mass (arrows), and postoperative (15 months) coronal (C) and sagittal (D) MRI scans with gadovist showing removal of the tumour. 
were negative. No mitosis was observed, and Ki67 positivity was $<1 \%$. Cytokeratinins 8 and 18 were positive.

At a clinical status examination performed 4 weeks after surgery, the patient experienced clinical improvement, but she still felt fatigue and had a tendency to palpitations. On suspicion that the patient had not been cured by the surgery, $30 \mathrm{mg}$ octreotide LAR was given i.m. every 4 weeks for another year. No arrhythmia persisted and the patient needed no further cardiologic controls or anti-arrhythmic treatment.

MRI performed 3 months after surgery showed no remaining pituitary tumour, and several follow-up MRI scans have shown unaltered results (Fig. 2). One year later when octreotide therapy had been terminated, scintigraphies using 111-indium-octreotide, flurodeoxyglucose (18F-FDG) or 68-Ga-DOTATOC (edotreotide) tracers showed no signs of activity outside the pituitary.

Both plasma serotonin and chromogranin A were normalised after surgery and stayed within the normal range (Fig. 3). Post-surgical evaluation of the pituitary function showed a normal pituitary-adrenal response, a normal pituitary-thyroid function and slightly reduced

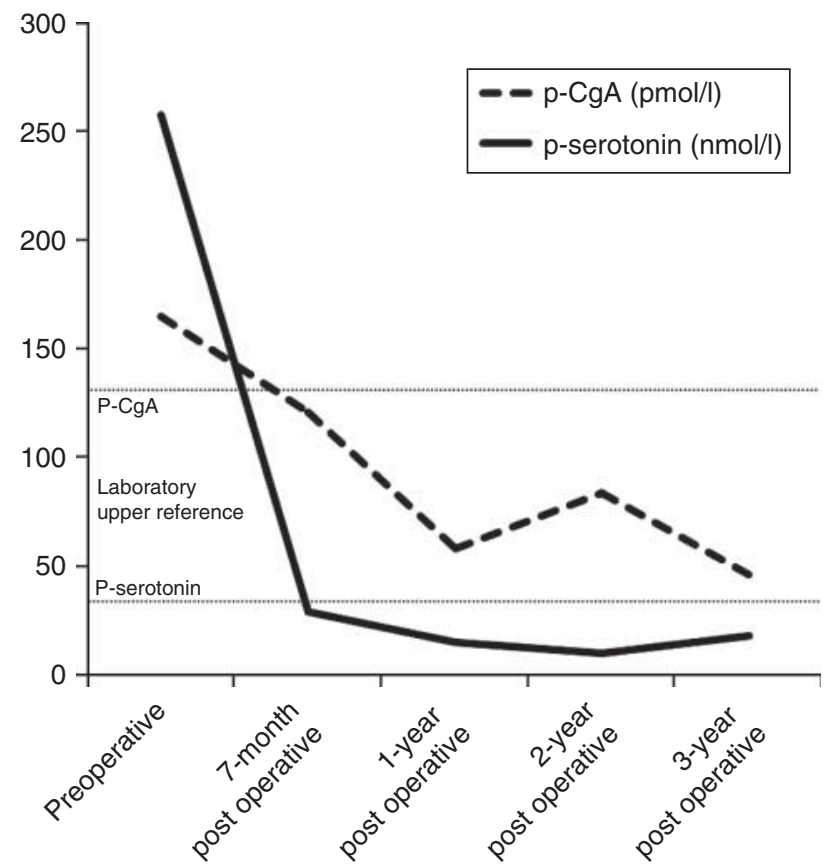

Figure 3

Preoperative and follow-up values of plasma serotonin and chromogranin A. Horizontal dotted lines indicate laboratory upper reference values for chromogranin $A(130 \mathrm{pmol} / \mathrm{l})$ and serotonin $(<30 \mathrm{nmol} / \mathrm{l})$.
GH secretion. The patient did, however, develop a partial diabetes insipidus. The pre-surgical visual field defect had been eliminated at the post-surgical follow-up.

\section{Discussion}

We report a case of a 56-year-old woman presenting years of symptoms compatible with carcinoid syndrome. Biochemical, nuclear imaging and clinical status before and after surgical therapy indicated that the syndrome was caused by a large serotonin-producing pituitary tumour.

Carcinoid syndrome is caused by an excessive production of mediators from a NET, most commonly serotonin (5). NETs are rare, slowly growing neoplasms with an incidence of 2.5 per 100000 women and 2.0 per 100000 men per year $(6,7,8)$. However, the number of patients presenting with a NET seems to be increasing (1). The most frequent site of origin is the bowel and gut, followed by the lungs $(1,6,7,8)$, whereas $10 \%$ seem to have another specific or unknown origin (6). We performed a PubMed search of English-language literature and found no prior reports of a primary NET in the pituitary. On the other hand, metastases to the pituitary from extra-pituitary NETs may occur in $0.9 \%$ of patient cases (9).

The diagnosis of NET is based on its clinical manifestations, biochemical measurements, highly specialised radiology and nuclear imaging. The most commonly used biochemical investigation is measurement of 24-h urinary excretion of 5-HIAA, a metabolite of serotonin. However, a proportion of patients with carcinoid syndrome have normal urinary excretion of 5-HIAA (5), as also seen in our patient in a single test. Studies of diagnostic tests in patients with carcinoid syndrome revealed that measurements of 5-HIAA in urine had a diagnostic sensitivity of $73 \%$ and a specificity of $100 \%$ (10). Thus, a negative 5-HIAA result in a patient with clinical suspicion of carcinoid syndrome should lead to measurement of serotonin in plasma, which is a more sensitive test (2). Also, chromogranin A is considered a valuable marker of both functioning and non-functioning NET with sensitivity up to $90 \%$ and specificity up to $100 \%$ (11). Biomarkers are useful not only for diagnosing but also for follow-up of patients after surgery.

Receptorscintigraphy using somatostatin analogues as receptor ligands is a useful diagnostic tool. The technique is based on the high expression of SSRs on NETs. Scintigraphy with 111-indium-marked octreotide has a sensitivity of more than $90 \%$, and is superior to CT/MRI in locating the primary tumour $(1,12,13)$. 
However, the sensitivity is lower for subtypes of tumours with low expression of sstr2 receptors or small lesions (14).

PET with 18F-FDG has limited sensitivity in the detection of well-differentiated NETs, but has particular importance as a prognostic marker (15). On the other hand, scintigraphy using 68-Ga-DOTATOC, which belongs to a newer generation of somatostatin analogues, has shown good results with high sensitivity (16).

Our patient had several symptoms that might suggest the presence of a carcinoid syndrome, but due to the dominance of cardiac and respiratory symptoms she was believed to suffer from a primary cardiac arrhythmia. The prolonged course with persisting symptoms and lack of clinical improvement despite therapy for arrhythmia even led to a psychological/psychiatric disorder being considered. Flushing was perceived as of menopausal origin, and was treated with oestrogen replacement therapy. In the setting of a carcinoid syndrome, it is not unusual that symptoms are misread before the correct diagnosis is given. Average diagnostic delay is up to 5-7 years from the initial onset of symptoms (3). The most common misdiagnosis for abdominal NET is irritable bowel syndrome (37\%), followed by food intolerance (18\%) and psychiatric disorders (17\%) (17). Five percent are misjudged to be caused by menopause (17).

The diagnostic breakthrough in our case was the biochemical testing of plasma levels of serotonin and chromogranin A. This led to a SST scintigraphy, which gave the diagnosis of an isolated pituitary/suprasellar mass. Notably, the normal pituitary as well as several pathologies in and around the sella may be visualised using such scintigraphy (18). Thus, the result of imaging has to be seen in the context of clinical and biochemical cure after the removal of the tumour. Histological examination including immunohistochemistry gave the appearance of a calcified null cell pituitary adenoma. There was no reaction for serotonin, but both plasma serotonin and chromogranin A normalised after the surgery and remained normal for the 3 years of follow-up (Fig. 3). Tumour content of synaptophysin was not studied. The benign histopathological appearance as well as the low Ki67 positivity (19) was compatible with the prolonged clinical phase with a gradual worsening of the condition over many years.

Initially, we speculated that the pituitary tumour might be a metastasis from an extra-pituitary primary NET. However, 3 years of observation and imaging, as well as biochemical testing after removal of the pituitary tumour, have given no support to this hypothesis. Based on clinical presentation, blood chemistry and imaging procedures, as well as improvement of the clinical condition and blood biochemistry after tumour removal, we conclude that the patient suffered from a carcinoid syndrome due to a serotonin-producing pituitary tumour. However, the possibility of an unknown primary NET or a spontaneously regressed NET outside the skull with a pituitary/suprasellar metastasis can in principle never be excluded.

\section{Conclusion}

We describe the first case of a pituitary adenoma leading to severe carcinoid syndrome.

Declaration of interest

The authors declare that there is no conflict of interest that could be perceived as prejudicing the impartiality of the research reported.

\section{Funding}

This research did not receive any specific grant from any funding agency in the public, commercial or not-for-profit sector.

\section{References}

1 Pape UF, Perren A, Niederle B, Gross D, Gress T, Costa F, Arnold R, Denecke T, Plöckinger U, Salazar R et al. ENETS Consensus Guidelines for the management of patients with neuroendocrine neoplasms from the jejuno-ileum and the appendix including goblet cell carcinomas. Neuroendocrinology 201295 135-156. (doi:10.1159/000335629)

2 O'Toole D, Grossman A, Gross D, Delle Fave G, Barkmanova J, O'Connor J, Pape UF \& Plöckinger U \& Mallorca Consensus Conference participants; European Neuroendocrine Tumor Society. ENETS Consensus Guidelines for the Standards of Care in Neuroendocrine Tumors: biochemical markers. Neuroendocrinology 200990 194-202. (doi:10.1159/000225948)

3 Modlin IM, Oberg K, Chung DC, Jensen RT, de Herder WW, Thakker RV, Caplin M, Delle Gave G, Kaltsas GA, Krenning EP et al. Gastroenteropancreatic neuroendocrine tumours. Lancet Oncology 2008 1 61-72. (doi:10.1016/S1470-2045(07)70410-2)

4 Srivastava A \& Hornick JL. Immunohistochemical staining for CDX.-2, PDX-1, NESP-55, and TTF-1 can help distinguish gastrointestinal carcinoid tumors from pancreatic endocrine and pulmonary carcinoid tumors. American Journal of Surgical Pathology 200933 626-632. (doi:10.1097/PAS.0b013e31818d7d8b)

5 Graham GW, Unger BP \& Coursin DB. Perioperative management of selected endocrine disorders. International Anesthesiology Clinics 200038 31-67. (doi:10.1097/00004311-200010000-00004)

6 Hemminki K \& Li X. Incidence trends and risk factors of carcinoid tumours. A national epidemiologic study from Sweden. Cancer 200192 2204-2210. (doi:10.1002/1097-0142(20011015)92:8<2204::AIDCNCR1564>3.0.CO;2-R)

7 Modlin IM \& Sandor A. An analysis of 8305 cases of carcinoid tumours. Cancer 199779 813-829. (doi:10.1002/(SICI)10970142(19970215)79:4<813::AID-CNCR19>3.0.CO;2-2)

8 Godwin JD. Carcinoid tumours: an analysis of 2837 cases. Cancer 1975 79 560-569. (doi:10.1002/1097-0142(197508)36:2<560::AIDCNCR2820360235>3.0.CO;2-4) 
9 Nassiri F, Cusimano M, Rotondo F, Horveth E \& Kovacs K. Neuroendocrine tumour of unknown origin metastasizing to a growth hormone-secreting pituitary adenoma. World Neurosurgery 201277 201.e9-201.e12. (doi:10.1016/j.wneu.2011.02.017)

10 Kwekkeboom DJ, Krenning EP, Lebtahi R, Komminoth P, Kos-Kudła B, de Herder WW \& Plöckinger U \& Mallorca Consensus Conference participants; European Neuroendocrine Tumor Society. ENETS Consensus Guidelines for the Standards of Care in Neuroendocrine Tumors: peptide receptor radionuclide therapy with radiolabeled somatostatin analogs. Neuroendocrinology 200990 220-226. (doi:10.1159/000225951)

11 Ferolla P, Faggiano A, Mansueto G, Avenia N, Cantelmi MG, Giovenali P, Del Basso De Caro ML, Milone F, Scarpelli G, Masone S et al. The biological characterization of neuroendocrine tumors: the role of neuroendocrine markers. Journal of Endocrinological Investigation $200831277-286$

12 Hage R, Brutel de la Rivière A, Seldenrijk CA \& Bosch JMM. Update in pulmonary carcinoid tumours: a review article. Annals of Surgical Oncology 200310 697-704. (doi:10.1245/ASO.2003.09.019)

13 Kaltsas G, Korbonits M, Heintz E, Mukherjee JJ, Jenkins PJ, Chew SL, Reznek R, Monson JP, Besser GM, Foley R et al. Comparison of somatostatin analog and meta-iodobenzylguanidine radionuclides in the diagnosis and localization of advanced neuroendocrine tumours. Journal of Clinical Endocrinology and Metabolism 2001862 895-902. (doi:10.1210/jc.86.2.895)
14 Balon HR, Brown TL, Goldsmith SJ, Silberstein EB, Krenning EP, Lang O, Dillehay G, Tarrance J, Johnson M, Stabin MG et al. The SNM practice guideline for somatostatin receptor scintigraphy 2.0. Journal of Nuclear Medicine Technology 201139 317-324. (doi:10.2967/ jnmt.111.098277)

15 Binderup T, Knigge U, Loft A, Federspiel B \& Kjaer A. ${ }^{18}$ F-fluorodeoxyglucose positron emission tomography predicts survival of patients with neuroendocrine tumors. Clinical Cancer Research 201016 978-985. (doi:10.1158/1078-0432.CCR-09-1759)

16 Menda Y, Ponto LL, Schultz MK, Zamba GK, Watkins GL, Bushnell DL, Madsen MT, Sunderland JJ, Graham MM, O'Dorisio TM et al. Repeatability of gallium-68 DOTATOC positron emission tomographic imaging in neuroendocrine tumors. Pancreas 201342 937-943. (doi:10.1097/MPA.0b013e318287ce21)

17 Toth-Fejel S \& Pommier RF. Relationships among delay of diagnosis, extent of disease, and survival in patients with abdominal carcinoid tumours. American Journal of Surgery 2004187 575-579. (doi:10.1016/ j.amjsurg.2004.01.019)

18 de Herder WW, Kwekkeboom DJ, Feelders RA, van Aken MO, Lamberts SW, van der Lely AJ \& Krenning EP. Somatostatin receptor imaging for neuroendocrine tumors. Pituitary 20069 243-248. (doi:10.1007/s11102-006-0270-5)

19 Nadler A, Cukier M, Rowsell C, Kamali S, Feinberg Y, Singh S \& Law CH $\mathrm{Ki}-67$ is a reliable pathological grading marker for neuroendocrine tumors. Virchows Archiv 2013462 501-505. (doi:10.1007/s00428-013$1410-8)$

Received 30 July 2013

Revised version received 28 October 2013

Accepted 30 October 2013 